\title{
PERCEPÇÃO DE USUÁRIOS DE PRAIAS EM RELAÇÃO AO LIXO MARINHO COMO UMA FERRAMENTA PARA AÇÕES EFETIVAS CONTRA ESSA PROBLEMÁTICA
}

\author{
BOM, F. C. ${ }^{1 *}$; NEVES, R. C. ${ }^{1}$; FRAGA, N. S. ${ }^{1}$; MUSIELLO-FERNANDES, J. ${ }^{1}$; \\ ZAPPES, C. A. ${ }^{12} \&$ SÁ, F. ${ }^{1}$ \\ 1. Universidade Federal do Espírito Santo, Departamento de Oceanografia e Ecologia - Vitória, \\ Espírito Santo, Brasil. \\ 2. Universidade Federal Fluminense, Instituto de Ciências da Sociedade e Desenvolvimento \\ Regional, Departamento de Geografia de Campos - Campos dos Goytacazes, Rio de Janeiro, Brasil.
}

*Autor correspondente: fabiocbom@gmail.com

\begin{abstract}
Bom, F. C., Neves, R. C., Fraga, N. S., Musiello-Fernandes, J., Zappes, C.A. \& Sá, F. (2020). Beach users' perception of marine litter as a tool for effective action against this problem. Braz. J. Aquat. Sci. Technol. 24(2). elSSN 1983-9057. DOI: 16312/bjast.v24n2. This study aimed to identify the perception of beach users about the problem of marine litter, and to characterize them from the socioeconomic point of view. To this, 43 open questionnaires were conducted with users from Curva da Jurema and Ilha do Boi beaches, in Vitória, Espírito Santo, southeastern Brazil. From the socioeconomic point of view, our results showed that there were no significant differences between the users of the two beaches. With respect to marine litter, the interviewees showed a high level of knowledge about this issue, knowing what the proper disposal of the litter they produce should be and what damage it can cause to the environment and also to humans. It is noteworthy that respondents blame other beach users for the accumulated litter, suggesting that the lack of education and awareness of other people is the main factor for beach garbage. Thus, we conclude that users of both beaches perceive the problem of marine litter, but do not identify their actions as part of the problem. Thus, constant measures are required in public-private partnerships focused on education on the subject, in addition to the installation of various points of garbage collection and monitoring their effectiveness. As a final product, actions were suggested with the different actors involved in the problem, in order to minimize the effects of this pollution.
\end{abstract}

Key Words: Environmental Education, Socio-environmental Oceanography, Marine Pollution, Coastal Management.

\section{INTRODUÇÃO}

O lixo marinho é definido como "qualquer material sólido persistente, manufaturado ou processado descartado ou abandonado no ambiente marinho e costeiro" (UNEP, 2009) e sua abundância nestes ecossistemas tem aumentado constantemente nas últimas décadas (Eriksen et al., 2014; Bergmann et al., 2015). Esse aumento causa sérios danos ecológicos e biológicos ao ambiente (Tourinho et al., 2010; Eerkes-Medrano et al., 2015) e também às atividades humanas, tanto do ponto de vista sanitário como socioeconômico (Oigman-Pszczol \& Creed, 2007; Cheshire \& Adler, 2009).

Do ponto de vista biológico, ocorre o emaranhamento de distintos organismos em redes fantasmas de pesca e plásticos manufaturados, além da ingestão destas partículas pelos animais, podendo ocasionar a mortalidade dos organismos e consequentes perdas na biodiversidade (Vegter et al., 2014; Eerkes-Medrano et al., 2015). Em uma perspectiva socioeconômica, o dano pode incluir o custo da degradação de bens e serviços do ecossistema, estéticos eeducacionais deáreas costeiras, como praias, bemcomo os riscos para a saúde humana (Ivar do Sul \& Costa, 2007; Galgani et al., 2013). Além disso, o lixo marinho também afeta uma ampla gama de atividades econômicas ligadas ao ambiente marinho, como a aquicultura, a pesca, a navegação e o turismo, com prejuízo ao seu uso recreativo, valor estético e graves perdas financeiras (Mouat et al., 2010; Galgani et al., 2019).

Nas últimas décadas houve um aumento significativo de estudos relacionados ao lixo marinho, identificando principalmente as causas deste problema e suas concentrações em ecossistemas costeiros e marinhos (Rochman etal., 2016). No entanto, por se tratar de ambientes complexos caracterizados por múltiplos interesses socioeconômicos, é também fundamental a participação da população, assumindo um papel essencial na gestão costeira (Marin et al., 2009).

A percepção ambiental, definida como um processo mental ao qual cada indivíduo sente o ambiente ao seu redor, valorizando-o em distintos níveis (Rio \& Oliveira, 1999; Coimbra, 2004), se tornou uma importante ferramenta para a avaliação de problemas ambientais em praias, uma vez que foi observada a importância do entendimento de como a comunidade se envolve com o meio ambiente em que habita, se engajando em possíveis ações mitigatórias (Timbó et al., 2019).

Löhr etal. (2017) identificaram que o envolvimento de usuários de praias na solução da problemática 
do lixo marinho pode ser uma eficiente ferramenta utilizada para diminuição deste problema nestes ecossistemas, porém pouco se sabe sobre esse tema (Veiga et al., 2016). Deste modo, se tornou essencial entender como a população percebe o problema de lixo marinho, possibilitando assim mudanças de atitudes e comportamento.

Mesmo sendo poucos ao redor do mundo, os estudos que avaliaram a percepção dos usuários de praias a respeito do lixo marinho resultaram no estabelecimento de soluções e ações efetivas a respeito dessa problemática, além de contribuir com valorosas sugestões para planos de manejo governamentais destes ecossistemas. Por exemplo, Campbell et al. (2016) identificaram que os usuários de praias da Tasmânia reconheceram que são os responsáveis pelo lixo marinho e devem ser os encarregados por limpá-lo, sendo a melhoria da educação como uma possível solução apontada para diminuir este problema. Já Rayon-Viña et al. (2018) sugerem em pesquisa realizada na Espanha que uma maior percepção a respeito do problema pode ser resolvida através do incentivo da limpeza das praias pela população local.

No Brasil, do mesmo modo, foram realizados estudos que objetivaram identificar a percepção de usuários de praias a respeito do lixo marinho. Os trabalhos pioneiros foram realizados por Santos et al.(2001; 2003) em praia do Rio Grande do Sul e mostraram pouco conhecimento dos usuários sobre os problemas ocasionados pelo lixo nos ambientes costeiros e marinhos. Este mesmo resultado foi também identificado por Santana-Neto et al. (2011) empraialocalizadanaBahia, mesmosendoentrevistados principalmente jovens vinculados ao ensino superior e por Magalhães \& Araújo (2012) em praia de Pernambuco. Por outro lado, houve também estudos que observaram um maior entendimento dos usuários de praias a respeito dessa temática, porém os autores observaram contradições quanto às respostas dadas pelos entrevistados e a elevada presença dos resíduos nas praias (Dias Filho et al., 2011; Timbó et al., 2019).

Deste modo, como essa problemática ainda não foi solucionada, são necessários mais estudos que contribuam com ações efetivas para a resolução desse problema, utilizando o conhecimento adquirido através dos usuários. No estado do Espírito Santo (ES), sudeste do Brasil, as praias da região metropolitana da Grande Vitória estão entre os principais atrativos turísticos (SETUR, 2017) e apesar do intenso uso, não se tem registro de estudos envolvendo a percepção de usuários sobre o lixo em praias nesta região.

Seguindo essa temática, o presente estudo teve como objetivo avaliar a percepção dos usuários de praias do município de Vitória, ES, em relação à poluição por lixo marinho encontrado nesses ambientes utilizados para o lazer. Como produto final, um box com sugestões de ações para os órgãos governamentais, setores privados e instituições de ensino e pesquisa foi elaborado, a fim de possibilitar um manejo adequado desses ecossistemas quanto ao lixo observado nas praias.

\section{MATERIAIS E MÉTODOS}

\section{Área de Estudo}

O Município de Vitória é a capital do Estado do Espírito Santo, localizado na região sudeste do Brasil, com uma extensão territorial de $2.331,01 \mathrm{~km}^{2}$ (IBGE, 2016) e população de 327.801 habitantes, conforme dados do censo de 2010 (IBGE, 2010). Tendo em vistaaimportância deste município, as praias da llha do Boi (20 18'30.97"S e 40 16'52.53"O) e Curva da Jurema (2018'32.57'S e 40¹7'17.64"O) foram escolhidas devido ao grande fluxo de pessoas, composto por moradores da região, por turistas e também por excursionistas (SETUR, 2017). A praia da llha do Boi é caracterizada por ter acesso mais restrito de pessoas, uma vez que não possui linhas de transporte urbano e o acesso dos usuários ocorre principalmente por veículos particulares, enquanto na Curva da Jurema o acesso é facilitado por meio do transporte público, presença de ciclovias e calçadão (Figura 1).

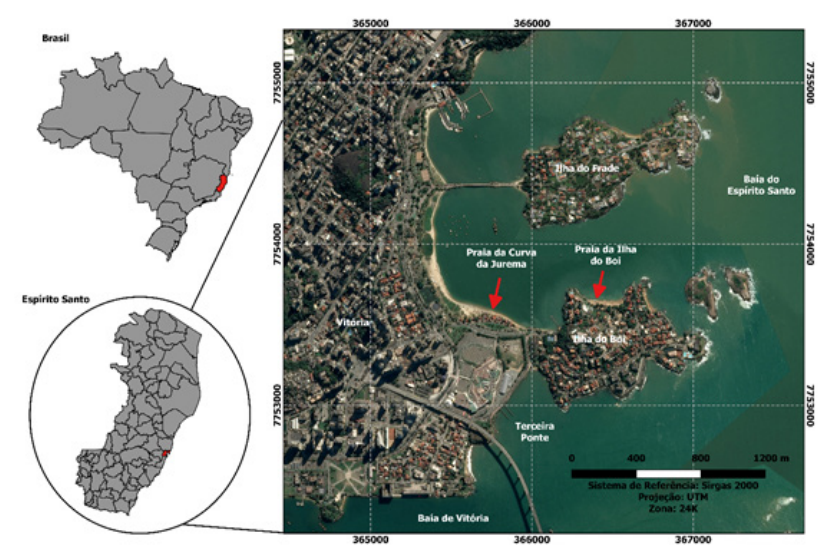

Figura 1: Praias do município de Vitória/ES em que foram realizadas as entrevistas (imagem retirada do Google Earth, 2019).

\section{Coleta de Dados}

Para a obtenção das informações foram realizados questionários que continham perguntas abertas, sendo flexível para adicionar relatos caso necessário (Schensul et al., 1999) (Tabela 1). A técnica de informação repetida em sincronia (o mesmo questionário aplicado a todos os entrevistados) foi utilizada para comparar as informações contidas nos relatos (Opdenakker, 2000). A primeira parte consistiu em caracterizar os entrevistados segundo 
seu perfil socioeconômico, de modo a avaliar possíveis diferenças entre os usuários das duas praias amostradas (Tabela 1). Para essa caracterização, foram realizadas perguntas a respeito do sexo, idade, renda familiar e nível de escolaridade do entrevistado, sendo pré-estabelecidas algumas categorias para cada uma dessas perguntas. Os entrevistados foram escolhidos de maneira aleatória em cada praia e a abordagem foi realizada de forma educada através da identificação dos entrevistados e explicação sobre os objetivos da pesquisa, como sugerido por Bastos (1999).

Tabela 1: Questionário aplicado aos usuários das praias da llha do Boi e Curva da Jurema, município de Vitória, estado do Espírito Santo.

\begin{tabular}{|c|c|}
\hline TÓPICOS & QUESTÕES \\
\hline & •Sexo \\
\hline & •Idade \\
\hline \multirow[t]{6}{*}{ Perfil dos Usuários } & -Escolaridade \\
\hline & •Renda \\
\hline & •Motivo de ir àquela praia \\
\hline & -Você considera essa praia suja em relação às \\
\hline & outras praias que conhece? \\
\hline & $\begin{array}{l}\text {-Que tipo de lixo você costuma produzir quando } \\
\text { frequenta a praia? }\end{array}$ \\
\hline \multirow{5}{*}{ Percepção do lixo } & $\begin{array}{l}\text {-O que você costuma fazer com o lixo que produz } \\
\text { na praia? }\end{array}$ \\
\hline & - Houve alguma vez em que você abandonou o lixo \\
\hline & $\begin{array}{l}\text { na praia? Se houve, por quê? } \\
\text {-Para você, quais as razões para haver lixo na praia? }\end{array}$ \\
\hline & -Que tipo de problema ou prejuízo o lixo na praia \\
\hline & $\begin{array}{c}\text {-Qual a sua sugestão para tentar diminuir a } \\
\text { quantidade de lixo na praia? }\end{array}$ \\
\hline
\end{tabular}

O sexo de cada entrevistado foi dividido em Homens e Mulheres. Quanto à idade se separou em seis categorias etárias: Até 20 anos; 21 a 30; 31 a 40; 41 a 50; 51 a 60; e 61 ou mais. A caracterização da renda familiar foi definida pelos seguintes critérios: Até dois salários mínimos; de 2 a 4; de 5 a 10; 11 a 20; e Acima de 20. Por fim, o nível de escolaridade foi dividido em 8 categorias: Analfabeto; Ensino Fundamental Incompleto; Ensino Fundamental Completo; Ensino Médio Incompleto; Ensino Médio Completo; Superior Incompleto; Superior Completo; e Superior com Pós. Os entrevistados não foram obrigados a responder às questões, sendo registrado Não-Informado quando optaram por não responder.

\section{Percepção sobre o Lixo Marinho}

Posteriormente à caracterização socioeconômica, os entrevistados foram abordados sobre questões relacionadas ao lixo marinho nas praias (Tabela 1). As perguntas realizadas foram baseadas em Santos et al. (1998), que realizaram estudo semelhante na Praia do Cassino, Rio Grande do Sul. Para não induzir os entrevistados às respostas, todas as questões foram realizadas de forma discursivas, possibilitando deste modo a obtenção de dados qualitativos a respeito do lixo marinho.

\section{Análise de Dados}

As respostas de cada pergunta foram organizadas em categorias de acordo com o questionário (Ryan \& Bernard, 2000), sendo todas as terminologias empregadas nas formas gráficas baseadas nas respostas obtidas com os entrevistados. A partir das informações contidas nestes gráficos foi possível descrever a percepção dos usuários de ambas as praias a respeito das questões levantadas. Ainda, foi utilizada a Análise de Discurso sobre os relatos a fim de compreender o contexto histórico e social dos entrevistados (Orlandi, 2012). Os relatos foram quantificados e suas frequências percentuais foram descritas. Testes de diferenças entre porcentagens foram realizados para avaliar possíveis diferenças significativas socioeconômicas entre os usuários das duas praias selecionadas para o presente estudo.

\section{RESULTADOS}

\section{Perfil dos Usuários}

Durante o estudo, 43 questionários foram realizados, sendo 25 na praia da Ilha do Boi e 18 na praia da Curva da Jurema. Entre os entrevistados foi identificada maior porcentagem de mulheres na Curva da Jurema $(61 \% ; n=11)$ e na llha do Boi $(64 \% ; n=16)$ e as faixas de idade dos entrevistados entre as praias apresentaram menor heterogeneidade na Curva da Jurema em relação à llha do Boi (Tabela 2). Não foi encontrada diferença significativa entre a renda dos usuários das duas praias $(p=0,39)$ apesar dos entrevistados da llha do Boi apresentarem renda mais elevada (Tabela 2). Com relação ao nível de escolaridade também não foi encontrada diferença significativa entre os usuários das duas praias $(p=0,18)$ sendo, no entanto, possível observar um maior grau de formação escolar entre os usuários da llha do Boi (Tabela 2).

Tabela 2: Perfil socioeconômico dos usuários das praias Curva da Jurema e Illha do Boi, Vitória, estado do Espírito Santo.

\begin{tabular}{|c|c|c|c|c|c|}
\hline & Illha do Boi & $\begin{array}{l}\text { Curva da } \\
\text { Jurema }\end{array}$ & & Ilha do Boi & Curva da Jurema \\
\hline SEXO & \multicolumn{5}{|c|}{ IDADE } \\
\hline Feminino & $16(64 \%)$ & $11(61 \%)$ & $\begin{array}{l}\text { Até } 20 \\
\text { anos }\end{array}$ & $6(24 \%)$ & - \\
\hline \multirow[t]{5}{*}{ Masculino } & $9(36 \%)$ & $7(39 \%)$ & $21-30$ & $5(20 \%)$ & $7(39 \%)$ \\
\hline & & & $31-40$ & $5(20 \%)$ & $7(39 \%)$ \\
\hline & & & $41-50$ & $2(8 \%)$ & $2(11 \%)$ \\
\hline & & & $51-60$ & $4(16 \%)$ & $2(11 \%)$ \\
\hline & & & $\begin{array}{l}61 \text { ou } \\
\text { mais }\end{array}$ & $3(12 \%)$ & - \\
\hline ESCOLARIDADE & \multicolumn{5}{|c|}{ RENDA } \\
\hline $\begin{array}{l}\text { Ensino Fundamental } \\
\text { Incompleto }\end{array}$ & $1(4 \%)$ & $1(5,5 \%)$ & Até 2 & $1(4 \%)$ & $2(11 \%)$ \\
\hline $\begin{array}{l}\text { Ensino Fundamental } \\
\text { Completo }\end{array}$ & - & $1(5,5 \%)$ & 2 a 4 & $8(32 \%)$ & $4(22 \%)$ \\
\hline $\begin{array}{l}\text { Ensino Médio } \\
\text { Incompleto }\end{array}$ & $3(12 \%)$ & $1(5,5 \%)$ & 5 a 10 & $6(24 \%)$ & $7(39 \%)$ \\
\hline Ensino Médio Completo & $1(4 \%)$ & $6(33 \%)$ & 11 a 20 & $6(24 \%)$ & $1(5,5 \%)$ \\
\hline Superior Incompleto & $5(20 \%)$ & $2(11 \%)$ & $\begin{array}{l}\text { Acima de } \\
20\end{array}$ & & $1(5,5 \%)$ \\
\hline Superior Completo & $9(36 \%)$ & $7(39 \%)$ & $\begin{array}{c}\text { Não } \\
\text { anformo }\end{array}$ & $2(8 \%)$ & $3(17 \%)$ \\
\hline $\begin{array}{l}\text { Superior com Pós } \\
\text { Não Informado }\end{array}$ & $\begin{array}{c}5(20 \%) \\
1(4 \%)\end{array}$ & - & & & \\
\hline
\end{tabular}


Ainda, foi possível identificar distintos motivos que induzem à visitação nas praias, sendo que na llha do Boi os entrevistados citaram principalmente 'tranquilidade' ( $48 \% ; n=12)$, 'limpeza' (28\%; n=7) e 'proximidade de suas moradias' $(24 \% ; n=6)$, enquanto na praia da Curva da Jurema é 'indicação pela internet/convite de alguém' ( $28 \%$; $n=5)$, 'maior infraestrutura com presença de quiosque' ( $17 \% ; n=3)$ e 'costume' em frequentar essa praia $(17 \% ; n=3)$.

\section{Percepção sobre Lixo Marinho}

No que tangue a percepção dos usuários das praias em relação à sujeira das praias que frequentam, houve elevada diferença nas respostas obtidas entre os entrevistados das duas praias, sendo que os frequentadores da praia da llha do Boi a consideram mais limpa que as demais (64\%), enquanto os usuários da praia da Curva da Jurema a consideram mais suja (61\%).

Quando questionados a respeito dos tipos de lixo que produzem ao ir à praia, os principais itens citados pelos entrevistados foram similares nas duas praias, estando relacionados principalmente com a alimentação humana, como latas, embalagens plásticas e palitos de picolé, além de produtos orgânicos, como coco e espiga de milho (Figura 2). Ainda, vale ressaltar o baixo número de entrevistados que consideram bitucas de cigarro como lixo $(8 \%$ dos entrevistados na Ilha do Boi e $22 \%$ na Curva da Jurema).

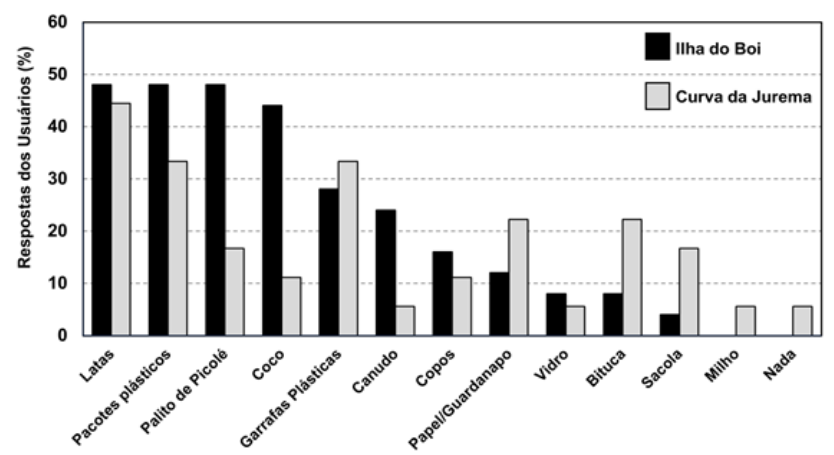

Figura 2: Tipos de lixo produzido pelos usuários das praias da llha do Boi e Curva da Jurema, município de Vitória, estado do Espírito Santo na percepção dos usuários.

Em relação ao destino dado ao lixo produzido pelos usuários das praias, a maioria relata principalmente o descarte em lixeiras públicas (80 e $67 \%$ dos entrevistados da Ilha do Boi e Curva da Jurema, respectivemente) ou o recolhimento e descarte em seus domicílios (48 e $28 \%$ ) e apenas um entrevistado declarou realizar a reutilização de resíduos produzidos, como garrafas plásticas (Figura 3). Além disso, houve um entrevistado que afirmou não produzir qualquer tipo de lixo quando frequenta a praia.

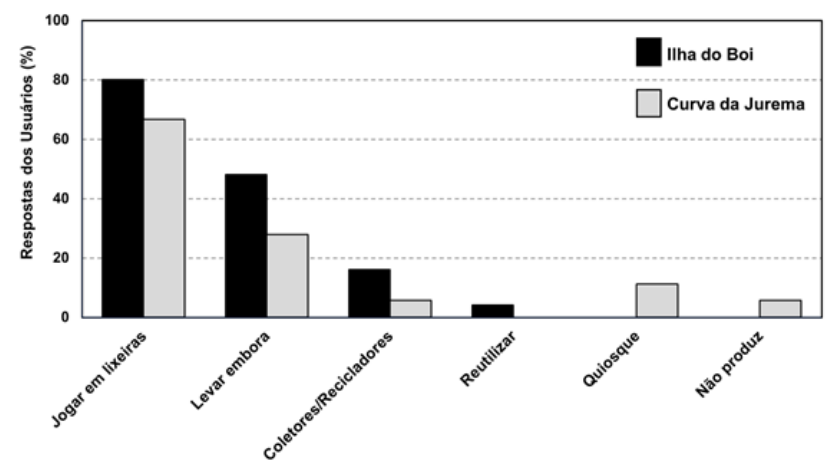

Figura 3: Destino dado ao lixo produzido pelos usuários da llha do Boi e Curva da Jurema.

Quando perguntados se houve alguma vez que abandonaram lixo na praia, a maioria dos entrevistados respondeu nunca terem deixado, sendo $68 \%$ e $56 \%$ dos entrevistados na llha do Boi e Curva da Jurema, respectivamente. Os demais usuários afirmaram terem deixado lixo na praia por diversos fatores, como esquecimento (16 e $6 \%$ ), falta de conscientização (8 e 11\%), falta de lixeiras (4 e $6 \%$ ) e preguiça (4 e 11\%). Ainda, houve um entrevistado da Curva da Jurema que afirmou deixar bitucas de cigarro quando vai à praia.

Quando verificado as razões de haver lixo nas praias, as respostas foram similares entre os entrevistados de ambas as praias, sendo a falta de educação/ética dos usuários das praias mencionado como o principal fator responsável por esse problema (com respostas de 60 e $72 \%$ dos entrevistados na llha do Boi e Curva da Jurema, respectivamente), seguido pelo descaso/preguiça e pela falta de informação das pessoas (Figura 4). Além dessas respostas, houve entrevistados que afirmaram que a razão de haver lixo na praia é devido à irresponsabilidade ambiental e falta de higiene da população, falta de lixeiras nas praias e falta de educação nas escolas. Houve também um entrevistado que culpou os turistas pela presença do lixo.

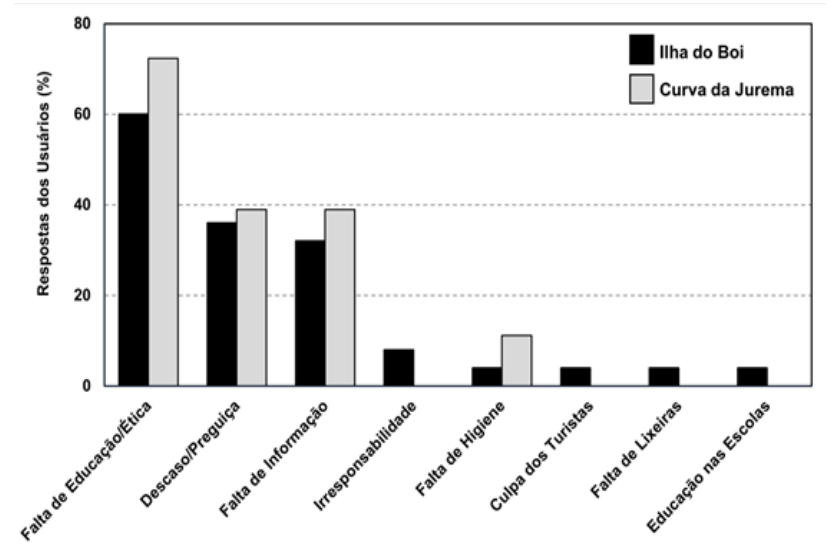

Figura 4: Motivos de haver lixo nas praias, segundo os entrevistados. 
Quando perguntados a respeito das consequências desta problemática, os entrevistados responderam principalmente que o lixo marinho pode causar problemas ambientais aos animais marinhos, como ingestão de plásticos por tartarugas, baleias e peixes (64 e 50\% dos entrevistados da llha do Boi e da Curva da Jurema, respectivamente, afirmando isso). Muitos entrevistados responderam também que o lixo marinho ocasiona poluição de mares e rios, formação de ilhas de lixo, causa mau odor e problemas aos humanos como interferência na saúde humana e alagamentos de cidades; além de interferência visual da paisagem e impacto negativo sobre o turismo, resultando em problemas econômicos à estas regiões (Figura 5).

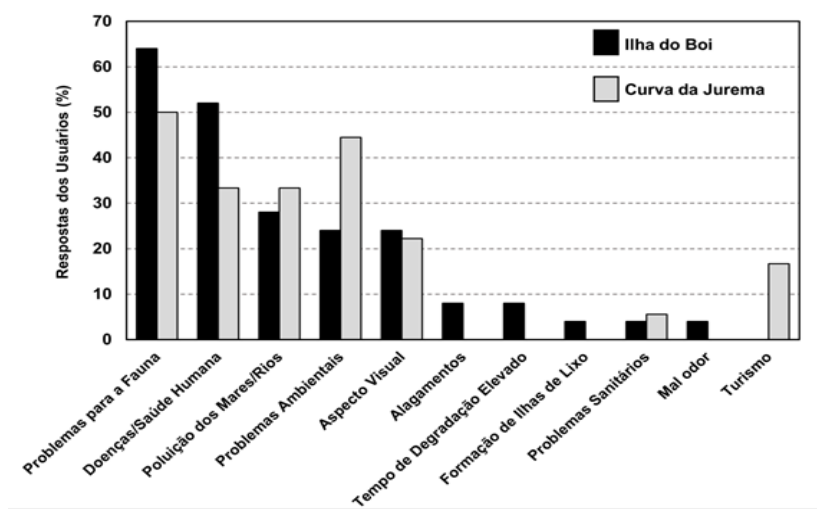

Figura 5: Principais problemas ocasionados pela presença de lixo marinho nas praias, segundo os entrevistados.

Ao todo, os entrevistados sugeriram 17 soluções para diminuição do lixo nas praias estudadas, sendo mais citadas ações voltadas para educação/informação dos usuários, aplicação de multas para quem descartar o lixo em local indevido, maior atitude governamental e maior publicidade através de propagandas, banners e placas informativas (Tabela 3).

Tabela 3: Soluções sugeridas pelos entrevistados para que haja uma diminuição do lixo nas praias.

\begin{tabular}{|c|c|}
\hline SOLUÇŐES DADAS PELOS ENTREVISTADOS & POSSIVEIS RESPONSÁVEIS \\
\hline •Destinação correta do lixo produzido & \\
\hline -Conferir outras utilidades para o lixo & Sociedade \\
\hline •Separação correta & \\
\hline -Educação Ambiental nas praias & $\begin{array}{l}\text { Governo, ONGs e institulçoes de } \\
\text { Pesquisa e Ensino }\end{array}$ \\
\hline $\begin{array}{l}\text {-Açõesucaçaao Ambiental em escolas } \\
\text { •campanhas de conscientização }\end{array}$ & \\
\hline •Multas & \\
\hline •Fiscalização & \\
\hline -Aumentar número de lixeiras em praias & Governo \\
\hline •Maior publicidade & \\
\hline -Aumentar número de catadores & \\
\hline $\begin{array}{l}\text { •Ações de contenção } \\
\text { - Quiosques coletarem o lixo produzido }\end{array}$ & \\
\hline $\begin{array}{l}\text {-Quiosques coletarem o lixo produzido } \\
\text {-Diminuir a produção de plásticos }\end{array}$ & Setor Privado \\
\hline •Substituição do plástico & \\
\hline a produção de materiais biodegradáveis & \\
\hline
\end{tabular}

\section{DISCUSSÃO}

Os resultados apresentados no presente estudo podem ser considerados como representativos a respeito da percepção a respeito do lixo marinho pelos usuários das praias de Vitória, uma vez que estudos sobre o tamanho amostral em Etnociências mostram que um intervalo entre 30 e 60 entrevistas são eficazes para o levantamento de informações a respeito de determinado estrato populacional (Mason, 2010; Morse, 1994). Apesar de não ter sido caracterizado nos questionários, através da Análise de Discurso dos entrevistados foi identificada uma maior presença de residentes das proximidades na praia da llha do Boi, o que já era esperado, devido o acesso ao bairro ser restrito principalmente pela ausência de transporte público. Diferentemente, na praia da Curva da Jurema houve maior relato de turistas/excursionistas, assim como identificado por Monteiro (2016) e Delgado (2018), evidenciando que esta praia possui um público mais variado, por ter fácil acesso e infraestrutura de quiosques. Porém, mesmo com essa distinta origem observada de parte do público entrevistado em cada praia, não houve diferença significativa na percepção a respeito do lixo marinho.

A limpeza da praia é identificada como um importante fator a ser levado em consideração para a escolha dos usuários em visitar determinada praia (Roca \& Villares, 2008; Marin et al., 2009; Lozoya et al., 2014) e no presente estudo foi possível observar que essa questão está relacionada possivelmente ao perfil social dos usuários de cada praia. Os entrevistados da Ilha do Boi demostraram um sentimento de pertença elevado, uma vezque parteéresidente das proximidades, relatando, portanto, ser uma praia limpa em relação às demais e que este é um importante fator por frequentarem essa praia. Por outro lado, os usuários entrevistados na Curva da Jurema não parecem ter entre as suas prioridades a limpeza da praia, mas principalmente as facilidades de infraestrutura local.

Quando perguntados a respeito dos tipos de lixo que encontram nas praias, os entrevistados deram respostas similares a outros estudos realizados em praias do Brasil, nos quais os itens alimentícios também foram os principais elementos citados pelos usuários (Santos et al., 2003; Santana-Neto et al., 2011; Fernandes \& Sansolo, 2013). Esta tipologia dos lixos observados em praias de uso recreacional com alta frequência no Brasil (Araújo \& Costa, 2003; Andrades et al., 2020) demonstra que são necessárias ações mais eficientes para mitigar essa problemática em caráter local. Este padrão observado no Brasil, similarmente ocorre nas praias de todo o mundo, sendo a maior produção de lixo nos ambientes costeiros composto principalmente por plásticos (garrafas, sacos e tampas), alumínio (latas e lacres) e vidro (garrafas), provenientes principalmente de atividades recreativas na costa (Bravo et al., 2009; Bergman et al., 2015, Kiessling et al., 2017). Esses materiais são de alta longevidade, de difíceis decomposições, persistindo 
por longos períodos no ambiente marinho, e podem ser transportados pelo oceano, poluindo outras regiões (Pegram \& Andrady, 1989). Outro aspecto relevante observado foi a falta de percepção quanto a possível reutilização dos materiais consumidos pelos usuários das praias, identificado por apenas um dos entrevistados. Esse fato deixa claro que a importância da busca pela reutilização de resíduos recicláveis gerados nas praias não está difundida entre os usuários entrevistados em ambas as praias abordadas, como também identificado por também identificado por Timbó et al. (2019), e deve ser mais contemplada em futuros planos de ação a respeito dessa temática.

A discrepância observada entre a quantidade de lixo presente nas praias, durante a realização as entrevistas, e o baixo relato de pessoas que deixam e/ou já deixaram lixo nas praias pode estar relacionada ao fato dos entrevistados não se sentirem à vontade para dizer sobre o próprio descarte incorreto dos seus resíduos, como observado por Magalhães \& Araújo (2012) em praia do Estado de Pernambuco. O fato da maioria das pessoas tenderem a não assumirem seus erros abertamente e culparem os outros como os responsáveis pelo lixo descartado incorretamente também pode ser um dos motivos, tendência também observada por Santos et al. (2005) em praia do Rio Grande do Sul. Santos et al. (2003) observaram este mesmo padrão, com $86,3 \%$ dos entrevistados atribuindo a presença de lixo nas praias à falta de conscientização dos demais usuários, indicando que não se sentem parte da problemática.

A respeito da percepção dos entrevistados sobre os problemas causados pelo lixo nas praias, as respostas foram similares às encontradas por Santana-Neto et al. (2011) e Santos et al. (2005) e evidenciam a baixa percepção dos usuários sobre os impactos do lixo na praia sobre o aspecto turístico e as suas possíveis perdas financeiras para a região. A intensa veiculação de informação nas diferentes mídias sobre as interferências do lixo marinho na fauna pode ser responsável pela detalhada percepção observada dos entrevistados sobre o assunto. Portanto, a partir dos relatos dos entrevistados, essas campanhas de associação do lixo à fauna podem ser consideradas como eficientes, permanecendo como importante ferramenta para sensibilização dos frequentadores das praias e elaboração de estratégias de conservação, principalmente por meio da utilização das diferentes redes sociais.

Quanto as bitucas de cigarro, estudos mostram que anualmente 6 trilhões de cigarros são consumidos em todo o mundo, e 4,5 trilhões são jogados no ambiente, sendo considerados a forma mais comum de lixo pessoal no mundo (Araújo \& Costa, 2019), assim como um dos itens mais encontrados em praias (Ivar do Sul et al., 2011; Rosevelt et al., 2013; Munari et al., 2016). Porém, no presente estudo poucos entrevistados relataram-nas como um tipo de lixo, como também identificado por Dias -Filho et al. (2011) e Timbó et al. (2019) em praias brasileiras e diferentemente de Rath et al. (2012) em entrevistas nos Estados Unidos e Kataržytè et al. (2020) em praias alemãs, que observaram que a maioria dos fumantes e não fumantes compreendiam que as bitucas de cigarro são um problema ambiental e apenas uma minoria de fumantes ainda não as reconhece como lixo.

Por fim, os entrevistados sugeriram diversas formas de mitigação contra o problema do lixo marinho, principalmente relacionadas à educação ambiental da população em geral, campanhas de conscientização, pagamento de multas, aumento do número de lixeiras nas praias e diminuição da quantidade de lixo produzido. Resultados semelhantes foram encontrados por Campbell et al. (2016) em praias da Tasmânia, Chen \& Teng (2016) em praia do Taiwan e Lucrezi \& Digun-Aweto (2020) em praias nigerianas, mostrando que possivelmente essas ações sirvam de orientação para as mudanças necessárias nestes ambientes, de forma a manter o ambiente da praia e a qualidade da recreação.

\section{CONSIDERAÇÕES FINAIS}

Através dos nossos resultados foi possível identificar que os usuários das praias da Curva da Jurema e llha do Boi, município de Vitória, ES possuem percepção seletiva a respeito do lixo marinho e suas interferências, com consciência parcial dos principais impactos gerados pelo descarte indevido de lixo para o meio ambiente e também para a qualidade de vida humana. Apesar dos entrevistados relatarem o correto descarte do lixo que produzem, foi observado uma elevada quantidade deste nas praias abordadas durante as entrevistas, sendo frequente o descarte irregular de lixo nestas praias e em outras abordadas na literatura. Ainda, são necessários extensos esforços nos ambientes para mudar essa percepção a respeito das bitucas de cigarro nas praias brasileiras, uma vez que é um dos tipos mais comuns de lixo encontrados e não há a identificação dos usuários das praias sobre ele.

Portanto, devido à complexidade do tema, se faz necessária a aplicação de ações articuladas por meio de parcerias entre órgãos governamentais, privados e instituições de ensino e pesquisa, a fim de atuarem em conjunto com a população para que esta se sinta parte do processo. Uma vez que várias propostas de mitigação foram testadas no mundo, mas até agora elas são relativamente isoladas e não possuem elevada efetividade na solução deste problema, os atores envolvidos precisam atuar de maneira integrada 
e coordenada na tomada de ações de prevenção, controle e mitigação de impactos causados pelo lixo marinho no ambiente costeiro. Por fim, com o auxílio das respostas fornecidas pelos entrevistados são sugeridas ações estratégias a fim de minimizar o descarte irregular de lixo em praias urbanas (Quadro 1).

Quadro 1: Ações estratégicas para possíveis soluções da problemática do lixo marinho.

\begin{tabular}{|c|c|c|c|c|}
\hline O quê? & Por que agir? & Como agir? & Quem? & Onde? \\
\hline $\begin{array}{l}\text { Prevenção do } \\
\text { descarte de } \\
\text { lixo no } \\
\text { ambiente }\end{array}$ & $\begin{array}{l}\text { Remoção de lixo no ambiente ocasiona } \\
\text { elevados custos para os órgãos } \\
\text { governamentais } \\
\text { Evitar impactos estéticos e sanitários no } \\
\text { ambiente costeiro } \\
\text { Evitar mortandade/contaminação dos } \\
\text { organismos marinhos }\end{array}$ & 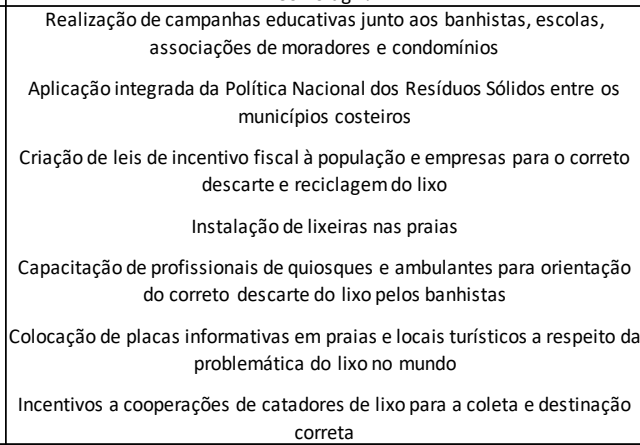 & $\begin{array}{c}\text { Secretaria de Gestão, } \\
\text { Planejamento e Comunicação } \\
\text { e Meio Ambiente } \\
\text { ONG's } \\
\text { Instituições de Ensino e } \\
\text { Pesquisa }\end{array}$ & $\begin{array}{c}\text { Escolas municipais, estaduais } \\
\text { e particulares } \\
\text { Praias, rios, áreas costeiras e } \\
\text { bairros dos munícipios } \\
\text { Empresas privadas }\end{array}$ \\
\hline $\begin{array}{l}\text { Remoção do } \\
\text { Lixo }\end{array}$ & \begin{tabular}{|c|}
$\begin{array}{c}\text { Minimizar os impactos estéticos e sanitários } \\
\text { aos frequentadores das praias }\end{array}$ \\
Diminuir os impactos nos organismos \\
marinhos \\
$\begin{array}{l}\text { Desenvolver sentimento de responsabilidade } \\
\text { pela manutenção do meio ambiente saudável } \\
\text { junto a população }\end{array}$ \\
\end{tabular} & $\begin{array}{c}\text { Ações de limpeza de praias e correta destinação do lixo envolvendo a } \\
\text { população e estudantes de escolas } \\
\text { Remoção periódica do lixo pelos municípios }\end{array}$ & $\begin{array}{l}\text { Secretaria do Meio Ambiente } \\
\text { e Educação } \\
\text { ONG's } \\
\text { Instituições de Ensino e } \\
\text { Pesquisa }\end{array}$ & $\begin{array}{l}\text { Praias, rios e áreas costeiras } \\
\text { Locais de descarte irregular } \\
\text { do lixo }\end{array}$ \\
\hline $\begin{array}{l}\text { Maior rigidez } \\
\text { na legislação }\end{array}$ & $\begin{array}{c}\text { Falta de legislação rígida não inibe o descarte } \\
\text { incorreto do lixo produzido pela população e } \\
\text { empresas }\end{array}$ & $\begin{array}{l}\text { Criação e revisão de leis e multas que inibam o descarte irregular do lixo } \\
\text { Fiscalização efetiva por parte dos órgãos governamentais nos municípios }\end{array}$ & $\begin{array}{l}\text { Poderes Legislativo e } \\
\text { Executivo Municipais }\end{array}$ & $\begin{array}{l}\text { Câmara de vereadores para } \\
\text { criação de leis } \\
\text { Prefeituras municipais para a } \\
\text { fiscalização e aplicação de } \\
\text { multas } \\
\text { Praias, rios e áreas costeiras }\end{array}$ \\
\hline $\begin{array}{l}\text { Disseminação } \\
\text { de } \\
\text { informações }\end{array}$ & $\begin{array}{l}\text { Pouco conhecimento da população em geral } \\
\text { a respeito dos impactos do lixo no meio } \\
\text { ambiente } \\
\text { Elevado alcance das mídias sociais como } \\
\text { veículo de informações para a população }\end{array}$ & $\begin{array}{l}\text { Criação de campanhas publicitárias sobre a importância do correto } \\
\text { descarte do lixo, impacto nos ambientes costeiros e animais }\end{array}$ & \begin{tabular}{|} 
Secretaria de Gestão, \\
Planejamento, Meio \\
Ambiente e Comunicação \\
ONG's \\
Empresas Privadas de \\
Comunicação \\
Instituições de Ensino e \\
Pesquisa
\end{tabular} & $\begin{array}{l}\text { Mídias Sociais (Facebook, } \\
\text { Instagram, Twitter, entre } \\
\text { outros) } \\
\text { Canais Abertos de Televisão, } \\
\text { Rádio e Jornais }\end{array}$ \\
\hline $\begin{array}{l}\text { Reutilização } \\
\text { de materiais } \\
\text { recicláveis }\end{array}$ & $\begin{array}{l}\text { Criação de novas possibilidade de fontes de } \\
\text { renda para população } \\
\text { Incentivar a reutilização de materiais } \\
\text { recicláveis pela população }\end{array}$ & $\begin{array}{l}\text { Realização de oficinas para o aprendizado, fabricação e comercialização de } \\
\text { produtos criados a partir de materiais reciclados } \\
\text { Incentivo às empresas de materiais recicláveis a desenvolver e divulgar a } \\
\text { população de como reutilizar os materiais após o seu uso }\end{array}$ & $\begin{array}{l}\text { Secretaria de Meio Ambiente } \\
\text { e Comunicação } \\
\text { Sociedade Civil Organizada } \\
\text { ONG's } \\
\text { Empresas Privadas }\end{array}$ & $\begin{array}{l}\text { Comunidades de baixa renda } \\
\text { e com maior índice de } \\
\text { desocupação } \\
\text { Associações culturais e } \\
\text { artesanato }\end{array}$ \\
\hline $\begin{array}{l}\text { Ações sobre } \\
\text { bitucas de } \\
\text { cigarro }\end{array}$ & $\begin{array}{l}\text { Falta de conhecimento da população a } \\
\text { respeito da problemática das bitucas de } \\
\text { cigarro descartadas no meio ambiente } \\
\text { Grande quantidade de fumantes na } \\
\text { população }\end{array}$ & $\begin{array}{c}\text { Realização de campanhas educativas nas praias, escolas e mídias sociais } \\
\text { Recolhimento das bitucas de cigarro descartadas incorretamente } \\
\text { Instalação de cinzeiros em locais públicos }\end{array}$ & $\begin{array}{c}\text { Secretaria de Meio Ambiente } \\
\text { e Comunicação } \\
\text { ONG's } \\
\text { Empresas Privadas } \\
\text { Instituições de Ensino e } \\
\text { Pesquisa }\end{array}$ & $\begin{array}{l}\text { Praias, rios e áreas costeiras } \\
\text { Locais de descarte irregular } \\
\text { do lixo }\end{array}$ \\
\hline $\begin{array}{l}\text { Revisão dos } \\
\text { processos do } \\
\text { setor } \\
\text { produtivo }\end{array}$ & \begin{tabular}{|c|}
$\begin{array}{c}\text { Crescente produção e descarte de materiais } \\
\text { com longa duração no ambiente ocasiona } \\
\text { elevados danos ecossistêmicos }\end{array}$ \\
Inefetividade atual das políticas de manejo \\
dos resíduos sólidos \\
Necessidade de geração de produtos menos \\
impactantes ao meio ambiente
\end{tabular} & $\begin{array}{l}\text { Incentivo a proibição/desuso do uso de sacolas plásticas e materiais de } \\
\text { uso único } \\
\text { Estímulo à produção e utilização de produtos duráveis pelas empresas, } \\
\text { população e comércio } \\
\text { Incentivo a novas tecnologias para produção de materiais menos } \\
\text { impactantes ao meio ambiente }\end{array}$ & $\begin{array}{l}\text { Poderes Legislativo e } \\
\text { Executivo Municipais } \\
\text { Empresas Privadas } \\
\text { Sociedade Civil Organizada } \\
\text { Instituições de Ensino e } \\
\text { Pesquisa }\end{array}$ & $\begin{array}{l}\text { Audiências Públicas } \\
\text { Ambientes de Discussão } \\
\text { sobre a problemática } \\
\text { (Universidades, Congressos, } \\
\text { Simpósios, Encontros) }\end{array}$ \\
\hline
\end{tabular}

\section{AGRADECIMENTOS}

FCB e FS agradecem a Fundação de Amparo à Pesquisa e Inovação do Espírito Santo (FAPES). NSF e JMF agradece a Coordenação de Aperfeiçoamento de Pessoal de Nível Superior - CAPES [cod 001]. CMZ agradece à Fundação Carlos Chagas Filho de Amparo à Pesquisa do Rio de Janeiro - FAPERJ [E-26/203.202/2016 e E-26/202.789/2019] e ao Conselho Nacional de Desenvolvimento Científico e 
BOM, F. C. et al., (2020). Percepção de usuários de praias em relação ao lixo marinho.

Tecnológico - CNPq [Processo 400053/2016-0] pelo financiamento às pesquisas.

\section{REFERÊNCIAS}

Andrades, R.; Pegado, T.; Godoy, B.S.; Reis-Filho, J.A.; Nunes, J.L.; Grillo, A.C.; Machado, R.C; Santos, R.G.; Dalcin R.H.; Freitas M.O.; Kuhnen, V.V.; Barbosa, N.D.; Adelir-Alves, J.; Albuquerque, T.; Bentes, B.; \& Giarrizzo, T. 2020. Anthropogenic litter on Brazilian beaches: Baseline, trends and recommendations for future approaches. Mar. Pollut. Bull. 151: 110842.

Araújo, M.C.B.; \& Costa, M.F. 2003.- Análise qualiquantitativa do lixo deixado na Baía de TamandaréPE-Brasil por excursionistas. Rev. Gest. Cost. Integr.- J. Integr. Coast Zone Manag. 3: 58-61.

Araújo, M.C.B.; \& Costa, M.F. 2019. A critical review of the issue of cigarette butt pollution in coastal environments. Environ. Res. 172: 137-149. DOI: https://doi.org/10.1016/j.envres.2019.02.005

Bastos, R.L. 1999. Ciências humanas e complexidades: projetos, métodos e técnicas de pesquisa; o caos, a nova ciência. EDUFJF, Juiz de Fora, 128p.

Bergmann, M.; Gutow, L.; \& Klages, M. 2015. Marine anthropogenic litter. Springer, London, 456p.

Bravo, M.; de los Ángeles Gallardo, M.; Luna-Jorquera, G.; Núñez, P.; Vásquez, N.; \& Thiel, M. 2009. Anthropogenic debris on beaches in the SE Pacific (Chile): Results from a national survey supported by volunteers. Mar. Pollut. Bull. 58(11): 1718-1726.

Campbell, M.L.; Slavin, C.; Grage, A.; \& Kinslow, A. 2016. Human health impacts from litter on beaches and associated perceptions: a case study of 'clean'Tasmanian beaches. Ocean Coast. Manage. 126: 22-30.

Campbell, M.L.; Slavin, C.; Grage, A.; Kinslow, A. 2016. Human health impacts from litter on beaches and associated perceptions: a case study of 'clean' Tasmanian beaches. Ocean Coast. Manage. 126: 22-30. DOI: http://dx.doi.org/10.1016/j. ocecoaman.2016.04.002

Carvalho-Souza, G.F.; Ogasawara, H.A.; Abrão-Oliveira, J.G.; Aguiar, L.G.P.A.; \& Barreto, G.S. 2012. A percepção de crianças sobre o lixo marinho: uma abordagem lúdica na popularização das ciências. Rev. Eletr. Mest. Educ. Amb. 42: 1-7.

Chen, C. L.; \& Teng, N. 2016. Management priorities and carrying capacity at a high-use beach from tourists' perspectives: A way towards sustainable beach tourism. Mar. Policy. 74: 213-219.

Cheshire, A.; \& Adler, E. 2009. UNEP/IOC guidelines on survey and monitoring of marine litter.
Coimbra, J.A.A., 2004. Linguagem e Percepçãoo Ambiental. Em: Curso de Gestão Ambiental. Manole, Barueri.

Delgado, A.T.P. 2018. O Turismo Litorâneo nas Cidades de Vitória, Espírito Santo, Brasile Bahia de Banderas, Nayarit, México. Dissertação de Mestrado, Universidade Federal do Espírito Santo, Vitória, ES, Brasil. 154p.

Dias Filho, M.; Silva-Cavalcanti, J.S.; Araújo, M.C.B.; \& Silva, A.C.M. 2011. Avaliação da percepção pública na contaminação por lixo marinho de acordo com o perfil do usuário: estudo de caso em uma praia urbana no Nordeste do Brasil. Rev. Gest. Cost. Integr.- J. Integr. Coast Zone Manag. 11(1): 49-55.

Eerkes-Medrano, D.; Thompson, R.C.; \& Aldridge, D.C. 2015 . Microplastics in freshwater systems: a review of the emerging threats, identification of knowledge gaps and prioritisation of research needs. Water Res. 75: 63-82. DOI: http://dx.doi. org/10.1016/j.watres.2015.02.012

Eriksen, M.; Lebreton, L.C.; Carson, H.S.; Thiel, M.; Moore, C.J.; Borerro, J.C.; Galgani, F.; Ryan, P.G.; \& Reisser, J. 2014. Plastic pollution in the world's oceans: more than 5 trillion plastic pieces weighing over 250,000 tons afloat at sea. PloS One, 9(12): e111913. DOI: https://doi.org/10.1371/journal. pone. 0111913

Fernandes, L.G.; \& Sansolo, D.G. 2013. Percepção ambiental dos moradores da cidade de São Vicente sobre os resíduos sólidos na Praia do Gonzaguinha, SP, Brasil. Rev. Gest. Cost. Integr.J. Integr. Coast Zone Manag. 13(3): 379-389. DOI: https://doi.org/10.5894/rgci416

Galgani, F.; Hanke, G.; Werner, S.D.V.L.; \& De Vrees, L. 2013. Marine litter within the European marine strategy framework directive. ICES J. Mar. Sci. 70(6): 1055-1064. DOI: https://doi.org/10.1093/ icesjms/fst122

Galgani, L.; Beiras, R.; Galgani, F.; Panti, C.; \& Borja, A. 2019. Impacts of marine litter. Front. Mar. Sci. 6: 208. DOI: https://doi.org/10.3389/ fmars.2019.00208

IBGE. 2010. Instituto Brasileiro de Geografia e Estatística - Censo Demográfico. Disponível em: https://cidades.ibge.gov.br.

IBGE. 2016. Instituto Brasileiro de Geografia e Estatística - Censo Demográfico. Disponível em: https://cidades.ibge.gov.br.

Ivar do Sul, J.A.; \& Costa, M.F. 2007. Marine debris review for Latin America and the wider Caribbean region: from the 1970s until now, and where do we go from here? Mar. Pollut. Bull. 54(8): 1087-1104. DOI: https://doi.org/10.1016/j. marpolbul.2007.05.004 
Ivar do Sul, J.A.; Santos, I.R.; Friedrich, A.C.; Matthiensen, A.; \& Fillmann, G. 2011. Plastic pollution at a sea turtle conservation area in NE Brazil: contrasting developed and undeveloped beaches. Estuar. Coasts. 34(4): 814-823. DOI: https://doi.org/10.1007/s12237-011-9392-8

Kataržytė, M.; Balčiūnas, A.; Haseler, M.; Sabaliauskaitè, V.; Lauciūtè, L., Stepanova, K.; Nazzari, C.; \& Schernewski, G. 2020. Cigarette butts on Baltic Sea beaches: Monitoring, pollution and mitigation measures. Mar. Pollut. Bull. 156: 111248.

Kiessling, T.; Salas, S.; Mutafoglu, K., \& Thiel, M. 2017. Who cares about dirty beaches? Evaluating environmental awareness and action on coastal litter in Chile. Ocean Coast. Manage.137: 82-95.

Krelling, A.P.; Williams, A.T.; \& Turra, A. 2017. Differences in perception and reaction of tourist groups to beach marine debris that can influence a loss of tourism revenue in coastal areas. Mar. Policy. 85: 87-99. DOI: http://dx.doi.org/10.1016/j. marpol.2017.08.021

Löhr, A.; Savelli, H.; Beunen, R.; Kalz, M.; Ragas, A.; \& Van Belleghem, F. 2017. Solutions for global marine litter pollution. Current opinion in environmental sustainability, 28: 90-99.

Lozoya, J.P.; Sardá, R.; \& Jiménez, J.A. 2014. Users expectations and the need for differential beach management frameworks along the Costa Brava: Urban vs. natural protected beaches. Land use policy. 38: 397-414.

Lucrezi, S.; \& Digun-Aweto, O. 2020. "Who wants to join?" Visitors' willingness to participate in beach litter clean-ups in Nigeria. Mar. Pollut. Bull. 155: 111167.

Magalhães, S.E.F.; \& Araújo, M.D. 2012. Lixo marinho na praia de Tamandaré (PE-Brasil): caracterização, análise das fontes e percepção dos usuários da praia sobre o problema. Trop. Oceanogr. 40(2): 193-208. DOI: https://doi. org/10.5914/tropocean.v40i2.5339

Marin, V.; Palmisani, F.; Ivaldi, R.; Dursi, R.; \& Fabiano, M. 2009. Users' perception analysis for sustainable beach management in Italy. Ocean Coast. Manage. 52(5): 268-277.

Mason, M. 2010. Sample size and Saturation in PhD studies using qualitative interviews. Forum Qualit. Social Res. 11: 3. DOI: http://dx.doi.org/10.17169/ fqs-11.3.1428

Monteiro, R.S.L. 2016. Territórios no espaço público: um estudo de caso nas praias de Vitória e Vila Velha-ES. Dissertação de Mestrado, Universidade Federal do Espírito Santo, Vitória. 154p.

Morse, J. M. 1994. Designing funded qualitative research. In: N. K. Denzin, \& Y. S. Lincoln
(Eds.) Handbook of qualitative research. Sage Publications Thousand Oaks, USA. 220-235 pp.

Mouat, J.; Lozano, R.L.; \& Bateson, H. 2010. Economic impacts of marine litter. Kommunenes Internasjonale Miljøorganisasjon.

Munari, C.; Corbau, C.; Simeoni, U.; \& Mistri, M. 2016. Marine litter on Mediterranean shores: analysis of composition, spatial distribution and sources in north-western Adriatic beaches. Waste Manage. 49: 483-490. DOI: https://doi.org/10.1016/j. wasman.2015.12.010

Oigman-Pszczol, S.S.; \& Creed, J.C. 2007. Quantification and classification of marine litter on beaches along Armação dos Búzios, Rio de Janeiro, Brazil. J. Coast. Res. 421-428.

Opdenakker, R. 2000. Advantages and disadvantages of four interview techniques in qualitative research. Forum Qualitative Sozialforschung/Forum Qualitative Social Research 7.

Orlandi, E.P. 2012. Análise do discurso. Princípios e Procedimentos. Pontes Editores, Campinas. 101p.

Pegram, J.E.; \& Andrady, A.L. 1989. Outdoor weathering of selected polymeric materials under marine exposure conditions. Polym. Degrad. Stabil. 26: 333-345. DOI: https://doi. org/10.1016/0141-3910(89)90112-2

Rath, J.M.; Rubenstein, R.A.; Curry, L.E.; Shank, S.E.; \& Cartwright, J.C. 2012. Cigarette litter: smokers' attitudes and behaviors. Int. J. Env. Res. Pub. He. 9(6): 2189-2203.

Rayon-Viña, F.; Miralles, L.; Gómez-Agenjo, M.; Dopico, E.; \& Garcia-Vazquez, E. 2018. Marine litter in south Bay of Biscay: Local differences in beach littering are associated with citizen perception and awareness. Mar. Pollut. Bull. 131: 727-735.

Rayon-Viña, F.; Miralles, L.; Gómez-Agenjo, M.; Dopico, E.; \& Garcia-Vazquez, E. 2018. Marine litter in south Bay of Biscay: Local differences in beach littering are associated with citizen perception and awareness. Mar. Pollut. Bull.131: 727-735.

Rio, V.D.; \& Oliveira, L., 1999. Percepção Ambiental: a Experiência brasileira. Studio Nobel, São Paulo.

Roca, E.; \& Villares, M. 2008. Public perceptions for evaluating beach quality in urban and semi-natural environments. Ocean Coast. Manage. 51(4): 314-329.

Rochman, C.M.; Cook, A.M.; \& Koelmans; A.A. 2016. Plastic debris and policy: using current scientific understanding to invoke positive change. Environ. Toxicol. Chem. 35: 1617-1626. DOI: https://doi. org/10.1002/etc. 3408

Rosevelt, C.; Los Huertos, M.; Garza, C.; \& Nevins, H.M. 2013. Marine debris in central California: 
BOM, F. C. et al., (2020). Percepção de usuários de praias em relação ao lixo marinho.

Quantifying type and abundance of beach litter in Monterey Bay, CA. Mar. Pollut. Bull. 71 (1-2): 299-306. DOI: https://doi.org/10.1016/j. marpolbul.2013.01.015

Ryan, G.; \& Bernard, H.R. 2000. Data management and analysis methods. In: Denzin, N.K.; Lincoln, Y.S. (org.) Handbook of Qualitative Research. Sage, London. 769-802pp.

Santana Neto, S.P.; Silva, I.R.; Cerqueira, M.B.; \& Tinôco, M.S. 2011. Perfil sócio-econômico de usuários de praia e percepção sobre a poluição por lixo marinho: Praia do Porto da Barra, BA, Brasil. Rev. Gest. Cost. Integr.- J. Integr. Coast. Zone Manag. 11(2): 197-206.

Santos, I.R.; Friedrich, A.C.; \& Duarte, E. 2003. Percepções sobre o lixo na praia do Cassino (RS, Brasil). Mundo Vida. 4(1): 11-17.

Santos, I.R.; Friedrich, A.C.; Wallner-Kersanach, M.; \& Fillmann, G. 2005. Influence of socio-economic characteristics of beach users on litter generation. Ocean Coast. Manage. 48(9-10): 742-752. DOI: https://doi.org/10.1016/j.ocecoaman.2005.08.006

Santos, I.R.D.; Friedrich, A.C.; Mariano, C.V.; Absalonsen, L., \& Duarte, E. 2001. Os problemas causados pelo lixo marinho sob o ponto de vista dos usuários da Praia do Cassino, RS. Ver. Eletr. Mestr. Educ.Ambient. 251-266.

Schensul, S.L.; Schensul, J.J.; \& Lecompte, M.D. 1999. Essential Ethnographic Methods: Observations, Interviews and Questionnaires. Altamira Press, Walnut Creek.

SETUR. 2017. Relatório de Pesquisa de Demanda Turística, Região Metropolitana. In: https:// observatoriodoturismo.es.gov.br/regioes-turisticas

Timbó, M.; da Silva, M.L.; de Oliveira Castro, R.; \& de Araújo, F.V. 2019. Diagnóstico da percepção ambiental dos usuários das praias de Itaipu e Itacoatiara quanto à presença de resíduos sólidos. Rev. Gest. Cost. Integr. J. Integr. Coast Zone Manag.19(3): 157-166.

Tourinho, P.S.; Ivar do Sul, J.A.; \& Fillmann, G. 2010. Is marine debris ingestion still a problem for the coastal marine biota of southern Brazil? Mar. Pollut. Bull. 60(3):396-401. DOI: https://doi.org/10.1016/j. marpolbul.2009.10.013

UNEP. 2009. Marine litter: A global challenge. Nairobi, Quênia.

Vegter, A.C.; Barletta; M.; Beck; C.; Borrero; J.; Burton; H.; Campbell; M. L.; Costa; M.F.; Eriksen; M.; Eriksson; C.; Estrades; A.; Gilardi; K.V.K.; Hardesty; B.D.; Ivar Do Sul; J.A.; Lavers; J.L.; Lazar; B.; Lebreton; L.; Nichols; W.J.; Ribic; C.A.; Ryan; P.G.; Schuyler; Q.A.; Smith; S.D.A.; Takada; H.; Townsend; K.A.; Wabnitz; C.C.C.; Wilcox; C.; Young; M.; \& Hamann L.C. 2014. Global research priorities to mitigate plastic pollution impacts on marine wildlife. Endanger. Species Res. 25(3): 225-247. DOI: https://doi.org/10.3354/esr00623

Veiga, J.M.; Vlachogianni, T.; Pahl, S.; Thompson, R. C.; Kopke, K.; Doyle, T.K.; Hartley, B.L.; Maes, T.; Orthodoxou, D.L.; Loizidou, X.I.; \& Alampei, I. 2016. Enhancing public awareness and promoting co-responsibility for marine litter in Europe: The challenge of MARLISCO. Mar. Pollut. Bull.102(2): 309-315.
Submetido: 04/2020

Revisado: 09/2020

Aceito:09/2020

Publicado: 09/11/2020 